infected with EBV. Furthermore, ELS-containing RA synovia transplanted into SCID mice supported production of ACPA and antiVCP1/VCP2 antibodies cross-recognised by ACPA. Analysis of $\mathrm{CD} 4+$ and $\mathrm{CD} 8+\mathrm{T}$-cell localisation and granzyme $\mathrm{B}$ expression suggests that EBV persistence in ELS-containing synovia is favoured by exclusion of $\mathrm{CD} 8+\mathrm{T}$ cells from $\mathrm{B}$-cell follicles and impaired CD8-mediated cytotoxicity.

Conclusions We demonstrated active EBV infection within ELS in the RA synovium that appears to contribute to local growth and differentiation of ACPA-reactive B cells.

\section{A6.6 THE ROLE OF SYNDECAN-4 IN EXPERIMENTAL COLITIS}

doi:10.1136/annrheumdis-2013-203220.6

'Mareike Fröhling, ${ }^{2}$ Dominik Bettenworth, ${ }^{1}$ Peter Paruzel, ${ }^{3}$ Frank Echtermeyer, ${ }^{2,4}$ Andreas Lügering, 'Thomas Pap, 'Athanasios Stratis. 'Institut of Experimental Musculoskeletal Medicine, University Muenster, 48149 Muenster, Germany; ${ }^{2}$ Department of Medicine B, University Muenster, 48149 Muenster, Germany; ${ }^{3}$ Department of Anesthesiology and Intensive Care Medicine, Medical University Hannover, 30625 Hannover, Germany; ${ }^{4} \mathrm{MVZ}$ Portal 10, Muenster, Germany

Background and Objectives The transmembrane heparan sulphate proteoglycan syndecan-4 ( $\mathrm{Scd} 4)$ has been implicated in cellmatrix adhesion, cell migration, differentiation, proliferation and plays an important role during inflammation in rheumatoid arthritis. Scd4 is a mediator and modulator of inflammatory signals, upon its binding of cytokines Scd4 acts either as a decoy receptor or through the initiation of Scd-dependend signalling, followed by the formation of a Scd4 complex. Cartilage damage is decreased in sdc4deficient mice, but osteopontin-mediated liver damage is increased. Because of these dual effects we investigate the impact of sdc4 in murine experimental colitis

Materials and Methods We performed DSS-induced colitis in Scd $4 \%$ and C57BL/6 WT mice. We used weight loss, colon length and histological scoring of colonic modifications to measure the course of colitis. Scd4 $\%$ and WT mice were orally gavaged with $5 \times 10^{8}$ colony-forming units (CFU) of invasive bacterium Citrobacter rhodentium (C. rhodentium). The changes of body weight and faecal excretion of $C$. rhodentium were monitored for 21 days followed by evaluation of histological changes after infection. The permeability of the colon was examined in vitro by infection of colon samples from Scd4 $\%$ and C57BL/6 WT mice with C. rhodentium. The migration behaviour of endothelial human cells (T-84) and scd4-siRNA T-84 knockdown cells was analysed by scratch assay

Results DSS-treated Scd4 $\%$ mice lost dramatically more body weight compared to the WT mice and the histological damage according to the Dieleman-Score was markedly increased. At day 19 of post infection the clearance of C. rhodentium in $\mathrm{Scd} 4 \%$ mice was markedly prolonged. In vitro infection of colon samples from Scd4 mice with $C$. rhodentium revealed a higher permeability for the bacterium compared to WT colon samples. The knockdown of Scd4 in human endothelial T-84 cells leads to delayed cell migration.

Conclusions Like in inflammatory liver damage, Scd4 appears to play an important role in colitis and exerts protective effects in intestinal inflammation. The Scd4 deficiency leads to a higher permeability of the colon to $C$. rhodentium and a delayed cell migration. Further analysis are needed to explore the mechanisms of Sdc4signalling in colitis.

\section{A6.7 ALTERATIONS IN NAILFOLD VIDEOCAPILLAROSCOPY IN PATIENTS WITH GRANULOMATOSIS WITH POLYANGIITIS (WEGENER'S): AN OBSERVATIONAL STUDY}

doi:10.1136/annrheumdis-2013-203220.7

Julia Uceda, Rosalía Martinez, María L Velloso, José L Marenco. Rheumatology Department, Valme University Hospital, Sevilla, Spain
Background Nailfold videocapillaroscopy (NFC), allows for the detection of changes in microcirculation. In the granulomatosis with polyangiitis (GPA) the existence of a defined pattern has not been found.

Objectives The main objective of our study was to detect the possible existence of a defined pattern in the microcirculation of the nailfold capillaries of patients with GPA. The second objective was to investigate the possible correlation between abnormalities found and systemic involvement

Methods We identified 10 patients with a current mean age of $55.7 \pm 16.5$ years and predominantly female $(60 \%)$. The mean age at diagnosis was 49.4 years. $70 \%$ had upper respiratory tract involvement, the same percentage had pulmonary involvement (cavitated nodules or alveolar haemorrhage), the cutaneous manifestations such as purpura or necrotic ulcers were present in $70 \%$. About $40 \%$ had renal involvement (renal failure, proliferative glomerulonephritis), and $40 \%$ had peripheral neurological involvement. NFC was carried out by the same rheumatologist, on fingers 3 through to 5 of both hands using a ZUZI videocapillaroscopy, trinocular, dual illumination and zoom of $1 \mathrm{X} 4 \mathrm{X}$.

Results Abnormalities of the microcirculation of nailfold capillaries were found in 8 of the 10 patients. Among the patients with this pathological microcirculation, $62.5 \%$ had structural alterations (tortuous capillaries), $50 \%$ presented with micro-haemorrhage (single or multiple), avascular areas were found in $37.5 \%$ and $75 \%$ showed lower capillary density. Neither capillary dilation nor the formation of new vessels were detected within the sample of patients.

Abstract A6.7 Table 1 Correlation between capilaroscopic finding with organ involvement

\begin{tabular}{lllllll}
\hline $\begin{array}{l}\text { Organ } \\
\text { involvement }\end{array}$ & $\begin{array}{l}\text { Pathological } \\
\text { capillaroscopy }\end{array}$ & $\begin{array}{l}\text { Abnormal } \\
\text { morphology }\end{array}$ & $\begin{array}{l}\text { Bleeding } \\
\text { Avascular } \\
\text { areas }\end{array}$ & $\begin{array}{l}\text { Reduced } \\
\text { capillary } \\
\text { density }\end{array}$ & Expansion \\
\hline Respiratory (7) & 5 & 3 & 3 & 2 & 5 & 0 \\
Renal (4) & 3 & 3 & 1 & 1 & 3 & 0 \\
Neurological (4) & 3 & 1 & 1 & 0 & 3 & 0 \\
Skin (7) & 6 & 3 & 3 & 2 & 4 & 0 \\
\hline
\end{tabular}

Conclusions We have observed, more frequent bleeding, avascular areas and reduced capillary density and these findings were not related with any specific organ involvement. There is one only study in GPA which communicates a high percentage of avascular areas. [1]

\section{Reference}

1. Anders HJ, Haedecke C, Sigl T, Krüger K. Avascular areas on nailfold capillary microscopy of Patients with Wegeners granulomatosis. Clin Rheumatol. 2000, 19(2):86-8.

\section{A6.8 BIOLOGICAL THERAPIES IN JUVENILE IDIOPATHIC ARTHRITIS}

doi:10.1136/annrheumdis-2013-203220.8

ML Velloso Feijoo, R Martinez Perez, J Uceda Montañes, JL Marenco de la Fuente. Rheumatology Unit, Valme University Hospital, Seville, Spain

Background Biological therapies have dramatically changed the prognosis for children with juvenile idiopathic arthritis (JIA). There are doubts about the possibility of discontinuing treatment once remission is achieved. We focus in this question in our series.

Objective To assess the efficacy and safety of these drugs in our series of patients with JIA.

Matherials and Methods We identified 9 children with JIA treated with biologic therapies, and we made a description of our experience.

Results The mean age was $14.55 \pm 5.85$, with a female predominance $(66.7 \%)$. At diagnosis, mean age was $4.94 \pm 2.9$, and at the beginning of biological treatment of $8.77 \pm 2.63$. The median time 
Abstract A6.8 Table 1

\begin{tabular}{llllllll}
\hline Patient & Sex & Age & Type & RF & ANA & Uveitis & Swollen joints \\
\hline 1 & Female & 10 & Systemic & Negative & Positive & No & Knee \\
2 & Female & 13 & Polyarticular & Negative & Negative & No & Knee and wrists \\
3 & Male & 10 & Systemic & Positive & Positive & No & Knee and wrists \\
4 & Female & 10 & Polyarticular & Negative & Negative & No & Temporomandibular and wrists \\
5 & Female & 11 & Polyarticular & Negative & Negative & No & Wrists and metatarsophalangeal \\
6 & Male & 7 & Oligoarticular & Negative & Negative & No & Ankles \\
7 & Male & 7 & Oligoarticular & Negative & Negative & No & Knees \\
8 & Female & 5 & Oligoarticular & Negative & Positive & Yes & Knees \\
9 & Female & 6 & Oligoarticular & Negative & Negative & No & Ankles \\
\hline
\end{tabular}

from diagnosis to initiation of biological therapy was $3.94 \pm 2.83$ years. The disease characteristics are detailed in the table.

All the children had previously received DMARDs (66.6\% methotrexate (MTX) and $33.3 \%$ MTX and sulfasalazine). Eight of the 9 patients $(88.9 \%)$ were taking corticosteroids at baseline. Eight received etanercept (ETN) and one Adalimumab (ADA), with good outcomes in all the patients unless 1 that had to switch from ETN to ADA due to inefficacy, and improved after the change. The steroids were suspended in $75 \%$ of children (6). Differences between mean values of CRP, ESR, and platelets from baseline to actual moment were statistically significant.

The median biologic time is 4 (1.11) years.

Actually all the children are in remission, two of them (patients 1 and 4) without biological treatment or classic DMARDs (since 5 and 2 years respectively).

None of the children have had significant adverse effects nor required hospitalisation from the beginning of therapy.

Discussion ETN has proved its efficacy in JIA (regardless of the type of onset), as it has been reported in multiple efficacy and safety studies, including long term studies of up to eight years of continuous therapy. $[1,2]$

We present our experience in children treated with up to 11 years, with good outcomes in terms of efficacy and safety in all the patients, and also 2 patients still in remission after 2 and 5 years without treatment.

\section{References}

1. EH Giannini et al, Long-Term Safety and Effectiveness of Etanercept in Children with juvenile idiopathic selected categories of arthritis. Arthritis Rheum. 2009, 60(9):2794-2804.

2. Pratsidou-Gertsi P, Trachana M, Pardalos G, Kanakoudi-Tsakalidou F. A follow-up study of juvenile idiopathic arthritis Patients with etanercept discontinued due to Who disease remission. Clin Exp Rheumatol. 2010 Nov-Dec, 28(6):919-22. Epub 2011 Jan 4.

\section{A6.9 DIRECTED TRANSPORT OF MICRORNAS FROM APOPTOTIC CELLS TO PHAGOCYTES BY MEMBRANE-COATED VESICLES (MCVS)}

doi:10.1136/annrheumdis-2013-203220.9

L Claßen, LO Tykocinski, F Wiedmann, S Krienke, A Lauffer, P Heyder, N Blank, M Schiller, HM Lorenz. Department of Internal Medicine V, Division of Rheumatology, University of Heidelberg, Heidelberg, Germany

Background and Objectives A distinctive feature of cell activation and apoptotic cell death is the formation of MCVs. MCVs have previously been identified as mediators of cell-to-cell communication and are recognised as carriers of microRNA. An impaired clearance of apoptotic debris has been observed in SLE patients. This is caused by an increased rate of apoptosis and by a defect in phagocytic-cell clearance.

We investigated differences in the microRNA content of MCVs released by activated and apoptotic lymphoblasts from normal healthy donors (NHDs) and SLE patients. MicroRNA content of lymphoblasts and MCVs and the effect of MCV uptake into monocytes were analysed.

Materials and Methods Activated lymphoblasts, UV-B irradiated lymphoblasts and corresponding MCVs of NHDs and SLE patients were compared in an Agilent microRNA array and validated by qPCR MiR-155 expression was determined by qPCR in monocytes with engulfed autologous UV-MCVs. Western blot was performed to investigate the expression of the miR-155 target protein Tab-2.

Results MiR-155, miR-155*, miR-34b and miR-99a were significantly less expressed in UV-lymphoblasts compared to nonirradiated lymphoblasts. The effect was even more pronounced in staurosporine-treated lymphoblasts. In contrast, the expression of miR-34a increased after UV-B irradiation but decreased under staurosporine treatment. The comparison of viable and apoptotic MCVs showed a decrease of miR-155* in apoptotic MCVs. In UV-MCVs, the miR-99a level was higher compared to viable MCVs. MiR-155 was not altered in MCVs after apoptosis induction. MiR-34a was expressed at higher levels in viable SLE lymphoblasts and MCVs compared to NHDs. In contrast, miR-34b expression was decreased in UV-lymphoblasts and UV-MCVs of SLE patients. In functional assays we could demonstrate higher miR-155 levels and consecutively decreased target protein levels in monocytes after engulfment of autologous UV-MCVs.

Conclusions Our data show an unequal distribution of the content of different microRNAs within apoptotic cells and cell derived MCV. This suggests a directional transport rather than a random distribution. Thus, cells can regulate their microRNA as well as the microRNA content within released MCV. We could show that microRNA and protein expression changes in phagocytes after UVMCV engulfment. Thus, our results suggest that MCVs could serve as a transport vehicle for microRNAs to mediate cell-cell communication and influence intracellular processes in the phagocyte. Disturbances of this system could contribute to the pathogenesis of SLE.

\section{A6.10 HIGH DISEASE ACTIVITY AND EROSION RATE IN SUDANESE RHEUMATOID ARTHRITIS PATIENTS}

doi:10.1136/annrheumdis-2013-203220.10

${ }^{1,2} \mathrm{Amir}$ I Elshafie, ${ }^{1,3}$ Sahwa Nourein, 'Vivek Anand Manivel, 'Azita Sohrabian, ${ }^{4}$ Mawahib IE Elidrisi, ${ }^{4}$ Elnour M Elagib, ${ }^{5}$ Musa AM Nur, ' Johan Rönnelid. 'Department of Immunology, Genetics and Pathology, Uppsala University, Uppsala, Sweden; '2Department of Pathology and Microbiology, Alribat University Hospital, Khartoum, Sudan; ${ }^{3}$ Khartoum Fertility Center, Khartoum, Sudan; "Rheumatology Unit, Alribat University Hospital, Khartoum, Sudan; ${ }^{5}$ Rheumatology Unit, Military Hospital, Omdurman, Sudan

Background and Objectives Reports from Nigeria claim that rheumatoid arthritis (RA) in Western Africa has relatively low disease activity with a 29 occurrence of hand erosions No data are published on RA in Sudan and our aim was to collect a first Sudanese RA cohort for comparative studies.

Materials and Methods 264 consecutive patients (87\% females) with RA according to the 1987 ACR criteria attending two 